\title{
Simulación de entorno 3D y cálculo a punto en radioterapia por procesamiento de imágenes diagnósticas
}

\section{Simulation environment 3D and calculation to point, in radiotherapy using diagnostic image processing}

\author{
Óscar Humberto Bernal- Romero \\ M.Sc. (c) Grupo de Óptica Moderna, \\ Universidad de Pamplona. Pamplona, Colombia \\ racsomed06@gmail.com
}

\author{
Martha Lucía Molina-Prado \\ Ph.D. Grupo de Óptica Moderna, \\ Universidad de Pamplona. Pamplona, Colombia \\ molinaprado@gmail.com
}

\author{
Néstor Alonso Arias- Hernández \\ Ph.D. Grupo de Óptica Moderna, \\ Universidad de Pamplona. Pamplona, Colombia \\ arias.her.nestor@gmail.com
}

\begin{abstract}
Resumen- El objetivo principal de la Radioterapia es suministrar una alta dosis de radiación ionizante al volumen definido como lesión o blanco, y reducir la dosis en los órganos o tejidos que por anatomía están cerca de ella, sin subdosificar la zona de tratamiento. Debido a esto, la visualización tridimensional de la zona a tratar, es de gran importancia en la simulación y la posterior planeación del tratamiento. Esta es una de las importancias que tienen las imágenes de diagnóstico médico (IDM) en este proceso.
\end{abstract}

La información visual de las Imágenes de Diagnóstico Médico (IDM) de estudios a pacientes con enfermedades Carcinogénicas, y que son obtenidas con equipos de diagnóstico (para este proyecto son de Tomografía Axial Computarizada TAC y de Resonancia Magnética Nuclear RMN), permiten extraer datos de gran importancia para el tratamiento. En este trabajo se obtiene la reconstrucción volumétrica (visualización 3D) de zonas tumorales de interés en las IDM, a través del procesamiento de las imágenes. La reconstrucción 3D de las zonas de interés permite determinar parámetros de simulación de entorno para tratamientos de teleterapia como la delimitación de zona a tratar, la reducción de zonas aledañas u órganos adyacentes. Lo anterior, posibilita obtener información para la focalización del haz de tratamiento, la determinación de tamaños de campos y las angulaciones de camilla, gantry y colimador. Con estos datos y los datos de calibración del acelerador (equipo de tratamiento), se determina el tiempo de tratamiento o cálculo a punto, lo cual permite en la Radioterapia mejorar el tratamiento y sus resultados.

Palabras clave - Imágenes diagnósticas, procesamiento de imágenes, simulación de entorno, teleterapia, dosimetría.

\begin{abstract}
The main goal of radiation therapy is to provide a high dose of ionizing radiation to the volume defined as injury or target, and reduce the dose to organs or tissues that are close to its anatomy, without subdosificar the treatment area. Due to this, the three-dimensional visualization of the treatment area is of great importance in subsequent simulation and treatment planning. This is a of the importance of using medical diagnostic images in this process. The information visual of medical diagnostic images done in studies carcinogenic ill patients are obtained with diagnostic equipment (for this project will focus on Computed Tomography CT and NMR Nuclear Magnetic Resonance), allow the acquisition of data that has great importance for treatment. By processing this group of images, the volume reconstruction is obtained (3D visualization) from tumor areas of each tissue or areas of interest, through of processing digital of images. The zones reconstruction 3D of interest permitted determining simulation parameters for teletherapy treatments as: the delimitation of area to be treated, reducing surrounding areas or organs. Additionally, it obtained the information for focusing of the treatment beam, for determining field sizes, angles of the couch, gantry and collimator. With these data and calibration data processing equipment (Accelerator), the treatment time or calculation point is determined, which allow in the Radiotherapy improve treatment and its results.
\end{abstract}

Keywords- Medical Diagnostic Images, image processing, simulation environment, teletherapy, dosimetry.

\section{INTRODUCCIÓN}

El descubrimiento de las energías ionizantes [1], fue uno de los grandes hallazgos a nivel cien- 
tífico que debeló la existencia de otras fuentes de energía y ramas de investigación, además de sus múltiples usos en diferentes áreas; ejemplo de ello, su utilización en la medicina como herramienta fundamental para la adquisición de las Imágenes de Diagnóstico Médico (IDM) [2], [3] $y$, a su vez, ha sido un apoyo fundamental e importante en los tratamientos y procesos de radioterapia, en el cual la radiación es utilizada como tratamiento [4]; estas dos últimas áreas son de interés para este trabajo.

Las IDM permiten visualizar las partes internas de un cuerpo sin necesidad de abrirlo, con ayuda de los equipos tomográficos más comúnmente usados, tales como el Tomógrafo Axial Computarizado (TAC) y el equipo de Resonancia Magnética Nuclear (RMN) [3]. Otros equipos que pertenecen al diagnóstico son la Gamma Cámara, el PET y los rayos $X$ convencionales [5]. Las IDM utilizadas en este estudio provienen de imágenes adquiridas por TAC y RMN, facilitadas por algunos centros de imágenes diagnósticas en Colombia.

La utilización de las IDM en la radioterapia permite visualizar la extensión de lesiones tumorales, como también los órganos en situación de riesgo o adyacentes a dicha zona. Estas imágenes al ser procesadas brindan información útil para determinar los parámetros de posicionamiento del equipo acelerador lineal [6], [7] y también los datos intrínsecos de las imágenes, como son las zonas de tratamiento en el paciente.

Los tratamientos de radioterapia son realizados con equipos robustos que permiten posicionar sus partes: Camilla, Gantry y Colimador. Lo anterior, para definir la mejor incidencia del haz de radiación hacia el interior del paciente donde se encuentra la lesión. Estos datos de posición son definidos por el radioterapeuta y el físico directamente en el equipo de tratamiento. El cálculo del tiempo de tratamiento es definido teniendo como referencia las IDM, las cuales permiten medir la profundidad de la lesión, y su tamaño define la apertura del haz de tratamiento (tamaño de campo); estos datos junto con los datos de calibración dosimétrica [8] del equipo de tratamiento facilitan definir el tiempo de irradiación diaria.

En este trabajo se presenta una visualización 3D de órganos o zonas tumorales junto con teji- dos y zonas sanas, con el objetivo de proporcionar al radioterapeuta un objeto de estudio más definido, para una visualización clara de la zona a tratar, evitando suministrar una dosis de radiación en lugares sanos y mejorar las condiciones de tratamiento, para luego realizar el cálculo a punto.

\section{DATOS: CALIBRACIÓN DISTANCIA INTERPIXEL}

El tratamiento de un paciente en Teleterapia inicia con la toma de imágenes diagnósticas; para este fin, es muy importante definir el posicionamiento del paciente, ya que debe ser reproducible a la hora del, debido a que al paciente se le suministra la dosis total del tratamiento en pequeñas secciones diarias.

Cada estudio tomográfico posee características propias, según la institución, el propósito y la técnica de adquisición de las IDM. Los datos presentados en este artículo son los obtenidos de un estudio de RMN contrastado de un equipo de 1.5 Teslas de marca General Electric (SIGNA EXCITE), el estudio digital presenta 19 imágenes del cráneo de un paciente con diagnóstico de cáncer cerebral. Facilitadas por la empresa Radioterapia del Norte Ltda.

En todo grupo de IDM, cada imagen presenta, además de la zona del cuerpo, datos personales del paciente, como también datos del equipo y parámetros usados en la adquisición de las IDM, en los cuales están una escala de magnificación para la imagen en sentido x y y, con su respectivo valor de distancia y también con valores del grosor del corte de cada imagen; y la distancia de separación entre cada una de ellas $\Delta z$. Los datos específicos del estudio utilizado son: $\mathrm{ST}=6 \mathrm{~mm}$, espesor del corte y SP $\Delta \mathrm{z}=7 \mathrm{~mm}$ separación entre las imágenes o cortes.

Las IDM en estudio presentan un tamaño original de 472 x 472 pixeles y una distancia interpixel de $\Delta \mathrm{x}$ y $\Delta \mathrm{y}=0.91 \mathrm{~mm}$, de esta forma, podemos establecer un ambiente virtual tridimensional con el grupo IDM apiladas, donde el ancho y alto de la imagen darán información en los sentidos x y y respectivamente, y el número de imágenes junto con su separación $\Delta \mathrm{z}$ darán información de la coordenada $\mathrm{z}$ como se muestra en la Fig. 1. 
Fig. 1. PILA DE IDM Y SU ESCALA

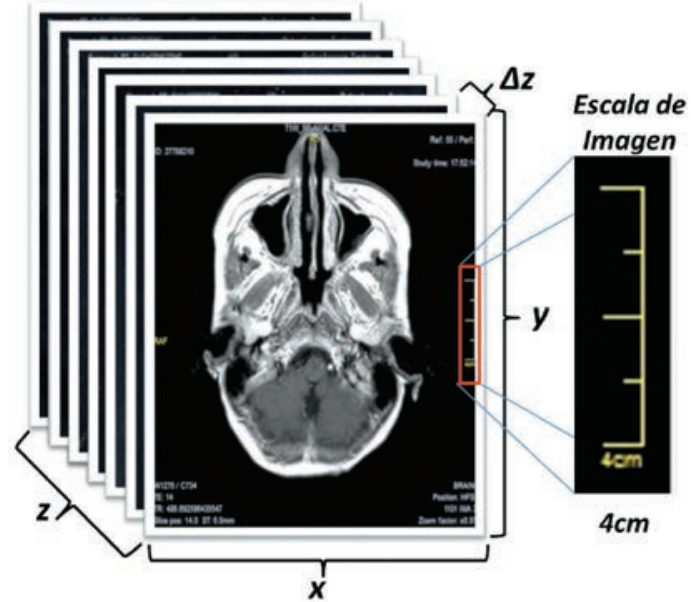

Fuente: autores

En algunos de los casos, el grupo de imágenes presenta información que no es de interés, la cual a tráves de un preprocesamiento es eliminada.

\section{PRE-PROCESAMIENTO DE LAS IDM}

Las IDM utilizadas para el desarrollo de este trabajo están en formato jpg, bmp, tiff y son imágenes digitales. El pre-procesamiento de las IDM de estudio se realiza con la herramienta Matlab. Una de las etapas de mayor dificultad es la segmentación de regiones de interés en imágenes médicas. Esto debido a la presencia de ruido y bajo contraste en zonas de importancia. Se ha realizado estudios comparativos de algoritmos que permiten eliminar el ruido [25]. Sin embargo, en esta ocasión las imágenes de trabajo son de bajo ruido por lo que la segmentación se realizó manipulando el histograma y a través de umbralización. Los niveles de gris en los pixeles de una IDM permiten ver diferencias entre los tejidos y órganos. La modulación del histograma de cada imagen [9],[10] ayuda a ganar definición en las áreas de interés, de tal forma que sobre ella, se realiza una binarización de la imagen. Después de este proceso, se utiliza un algoritmo morfológico con el fin de completar zonas de interés, luego se umbraliza logrando extraer los bordes de la zona de interés. Todo este proceso se desarrolla con el fin de definir completamente el perímetro de la zona del cuerpo, el cual en algunos casos hay que realizar con la intervención del usuario y con ayuda del algoritmo morfológico de llenado, obteniendo así la máscara. Esta última depende del tamaño de la forma seleccionada o zona de interés.

La máscara obtenida al ser multiplicada por la imagen inicial permite visualizar la información del cuerpo únicamente, teniendo como resultado una imagen libre de información no deseada a la cual Ilamaremos imagen limpia, este proceso se ilustra paso a paso en la Fig. 2. Es de aclarar que dicho proceso debe ser iterativo para cada una de las 19 imágenes del estudio.

Fig. 2. PASOS PARA EL PROCESO DE LIMPIADO DE IDM

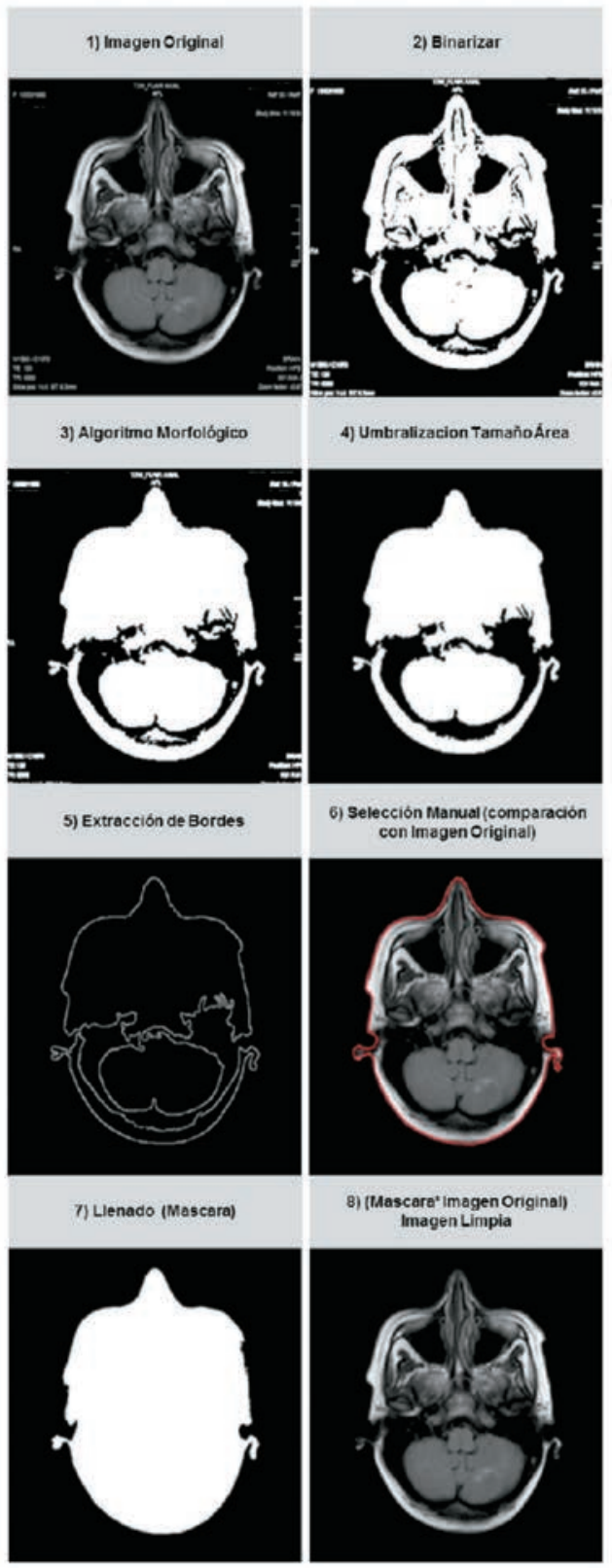

Fuente: autores. 


\section{PROCESAMIENTO DE LAS IMÁGENES}

Hasta este momento solo se ha dejado la información de interés en cada una de las imágenes a tratar en el presente trabajo. Al igual que en el pre-procesamiento, la herramienta utilizada para esta parte es Matlab.

\subsection{Extracción de contornos}

La información que se extrae en esta etapa serán las coordenadas de los pixeles límites entre los valores 0 y 1 [11], [12] de la máscara obtenida en el proceso anterior. Se debe recordar que la imagen sobre la cual se está trabajando es binaria. Para la extracción de los bordes de la máscara se utilizó el método Canny [13] que presenta mejores resultados comparado con algunos algoritmos de detección de bordes tradicionalmente usados, como Sobel, Prewitt, Roberts. Una vez aplicado el algoritmo para detección de bordes, se encuentran las coordenadas de dichos pixeles dentro de la imagen, los cuales representarán la parte externa del cuerpo (piel del paciente) (Fig. 3). Las IDM son imágenes axiales de la zona a visualizar, este proceso también es iterativo para el estudio tomográfico.

Fig. 3. DATOS SELECCIONADOS COMO PIEL

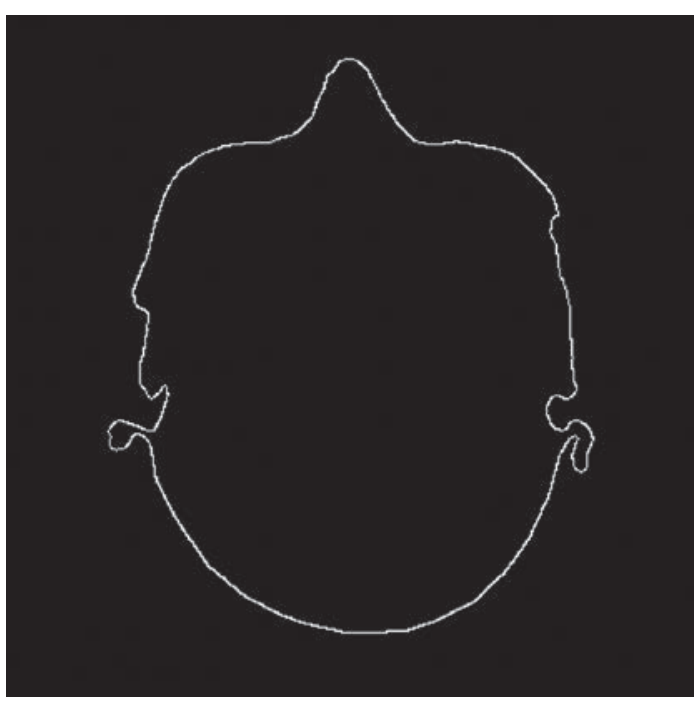

Fuente: autores.

\subsection{Visualización en 3D}

Teniendo cada uno de los contornos de las 19 imágenes con sus respectivas coordenadas y los factores de calibración $\Delta \mathrm{x}, \Delta \mathrm{y}$ y $\Delta \mathrm{z}$, es posible visua- lizar en 3D la información del cuerpo, lo cual permitirá además de la visualización, la posibilidad de hacer mediciones en cualquier sentido, brindando una mayor ventaja que un (Terapy Planing Sinstem TPS 2D) sistema de planeación en 2D. Cualquier TPS en 2D está restringido a mediciones bidimensionales con respecto a un punto, mientras que en el caso de las imágenes aquí tratadas, permite obtener información 3D, pues cada imagen es similar a la información de un plano del cuerpo. Como se ve en la Fig. 4, de esta forma se puede definir volúmenes y dar valores de distancia entre ellos.

Fig. 4. RECONSTRUCCIÓN 3D DE LOS DATOS DE PIEL

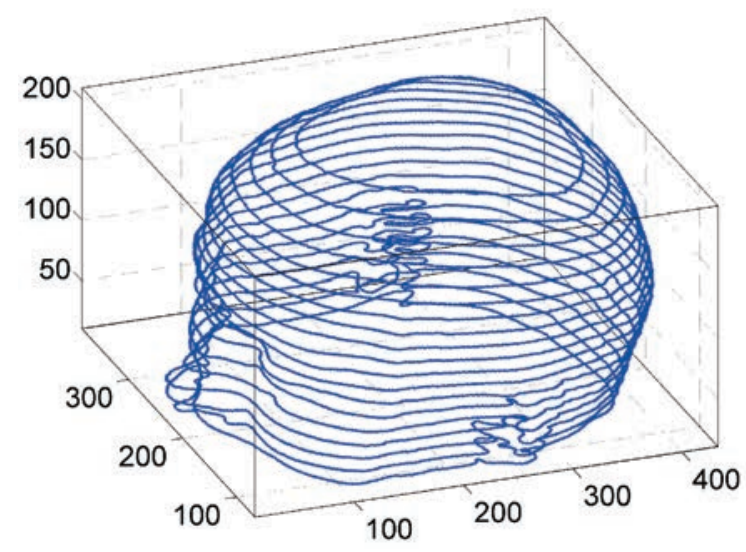

Fuente: autores.

\subsection{Definición de órganos y zonas de interés}

En términos de prácticas clínicas, estos pasos a seguir se limitan única y exclusivamente a los radioterapeutas, puesto que ellos toman la decisión según su criterio y el diagnóstico del paciente, delimitan las zonas que serán de gran importancia a la hora del tratamiento, donde la zona blanco o lesión será el volumen de mayor interés, seguido por cada uno de los tejidos u órganos adyacentes a esta zona [14], [15], y su reconstrucción permite obtener información de la distancia a la cual se encuentra del punto de tratamiento, dando el dato necesario para determinar la cantidad de radiación que recibirá cada uno de estos volúmenes a la hora de realizar el tratamiento de radioterapia.

Existen dos métodos utilizados en la prácti$\mathrm{ca}$, uno de ellos es manual y permite definir zonas según el criterio de quien realice el proceso y es iterativo para cada imagen; mientras que el segundo método es completamente digital, este 
proceso es más rápido, pero no siempre se obtiene los resultados esperados, por lo cual los dos métodos son complementarios e indispensables. El primer caso es utilizado cuando el órgano, tejido o masa tumoral no es claramente diferenciable o presenta una pequeña diferencia en escala de grises (bajo contraste), como se ve en Fig. 5a. Es de gran importancia recordar, aunque no es parte de este trabajo, que estas imágenes son con las que cuentan en su mayoría los pacientes, por su bajo costo y porque no pueden acceder a estudios tomográficos específicos para radioterapia.

Fig. 5a. DIFERENCIA ENTRE IMAGEN SIN CONTRASTE

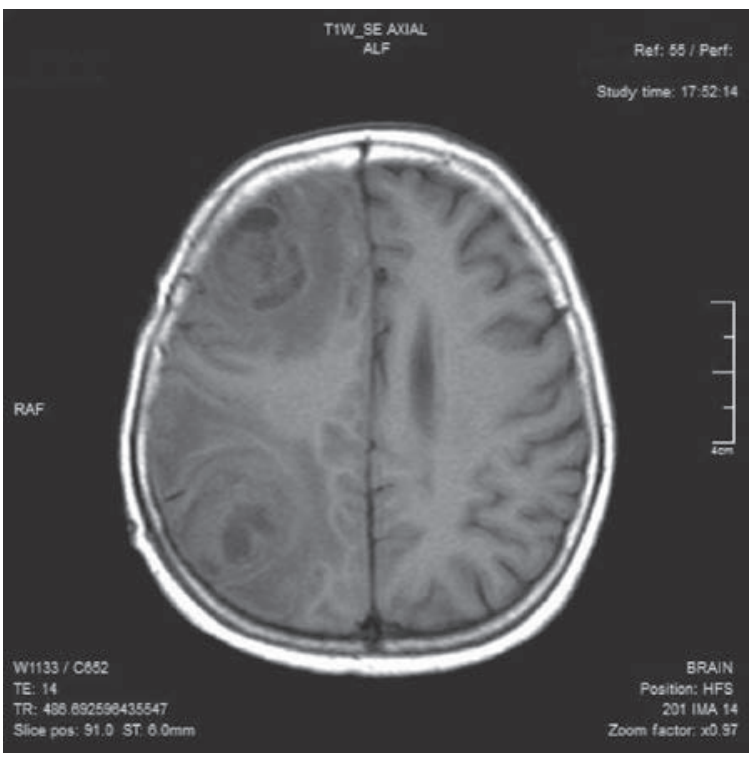

Fig. 5b. CON CONTRASTE

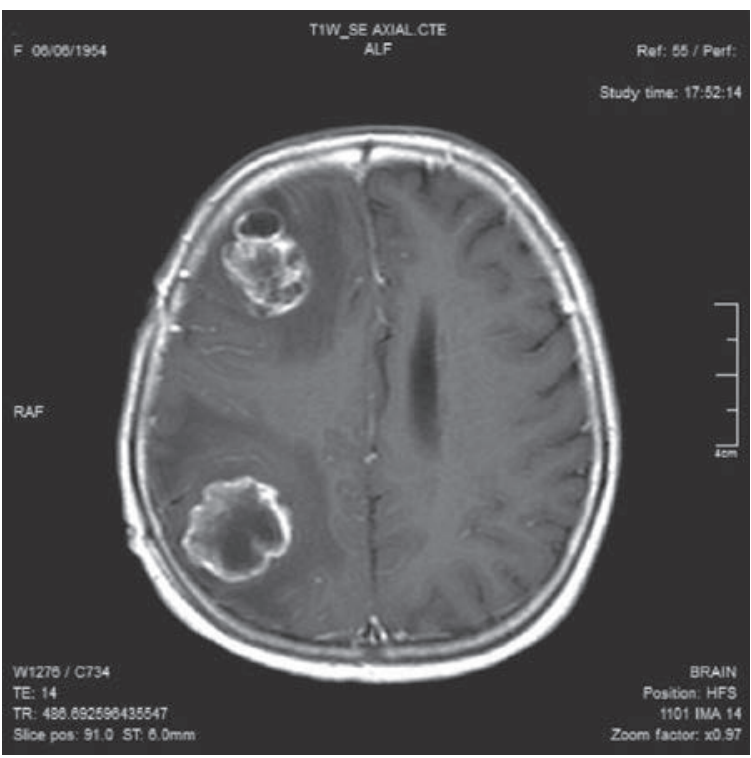

Para lograr una buena definición de las zonas de interés, en algunos de los casos, al adquirir las IDM se debe administrar al paciente un medio de contraste [2], [16],[17] que permitirá definir zonas de acuerdo al interés del estudio; a este grupo de imágenes se les conoce con el nombre de imágenes contrastadas (ver Fig. 5b) y son las utilizadas para adquirir los datos presentados en este trabajo por sus características.

Realizando nuevamente los procesos de la etapa de pre-procesamiento, pero restringiendo el número de imágenes, solo a las imágenes que contengan información de la lesión, se aplican la binarización, umbralización y el algoritmo morfológico de relleno por dilatación, obteniendo la máscara, que al ser multiplicada con la imagen limpia (obtenida en la etapa de pre-procesamiento), únicamente quedará la información de la zona interna del área seleccionada, y así se extraen las coordenadas de este grupo de datos del contorno en cada imagen que contenga información de la lesión. Ya que estas dan su ubicación dentro del cuerpo y su tamaño, permitiendo su representación tridimensional. Este algoritmo es necesario realizarlo iterativamente de acuerdo a los volúmenes de interés.

El segundo caso es el proceso digital de reconstrucción de zonas de interés, el cual se realiza con mayor rapidez en imágenes contrastadas, para este proceso es necesario trabajar con la pila de imágenes y limitar el tamaño del área de trabajo, para este procedimiento se creó una interfaz gráfica (Fig. 6a) que permite realizar el proceso de limitación del volumen a trabajar, en el cual se crea una caja que contenga todos los datos del estudio tomográficos y de forma iterativa se disminuye las dimensiones de cada uno de sus lados, hasta lograr disminuir su tamaño, de tal forma que esta nueva caja de trabajo queda dentro del conjunto de la piel y a su vez dentro de ella queda la totalidad de los pixeles que por criterio forman parte del volumen de interés y una menor cantidad de tejido adyacentes a él (Fig. 6b). En adelante, esta caja o volumen de interés se llamará limitbox. El paso a seguir es definir parámetros de umbralización dentro del limitbox, permitiendo así aplicar una vez más el proceso de binarización al conjunto y obtener la información del volumen para extraer las coordenadas de los pixeles que lo conforman. 
Los datos obtenidos hasta el momento permiten visualizar una imagen en tres dimensiones que contiene la piel de la zona del cuerpo en estudio y también los órganos delimitados, de esta forma, se tiene una amplia visualización de la morfología de los volúmenes de interés cercanos a la lesión (Fig. 7), lo cual es de gran ayuda para el radioterapeuta, permitiéndole prevenir o reducir efectos de la radiación en zonas adyacentes a la lesión durante el tratamiento.

\section{DETERMINACIÓN DE PARÁMETROS DE SIMULACIÓN Y DE CÁLCULO}

Los parámetros a determinar se dividen en dos grupos: parámetros de simulación de entorno que corresponden a las angulaciones de las partes del equipo de tratamiento (Camilla, Gantry y colimador), los cuales definen una dirección y sentido de ataque del haz de tratamiento con respecto a un punto; y los parámetros de cálculo de tiempo de tratamiento, los cuales dependen de la dirección del haz y son la profundidad a la que se encuentra la lesión y el tamaño de campo de tratamiento. Estos dos tipos de parámetros se deben referenciar a un punto en el espacio el cual se define como el isocentro de la lesión o punto de cálculo en la Fig. 7 representado por T1. A continuación, se definen los parámetros de simulación al igual que los parámetros de cálculo.

\subsection{Parámetros de simulación}

\subsubsection{Isocentro o punto de cálculo}

La visualización de las zonas de interés es de gran importancia para la determinación de los parámetros de simulación de entorno. Es necesario tener un punto referencia en el espacio para la determinación de estos parámetros.

A cada uno de los volúmenes limitados se les extrae las coordenadas de los pixeles que lo conforman. Aplicando la ecuación de centroíde tanto para x, y y z (1), es posible establecer las coordenadas del punto central en cada uno de los volúmenes definidos, como se ve en la Fig. 8.

$$
X_{c m}=\frac{\sum_{i} x_{i}}{N}
$$

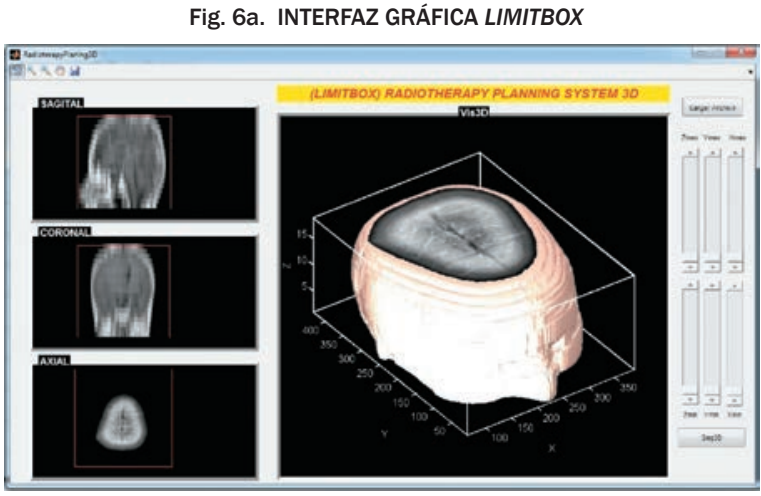

Fuente: autores.

Fig. 6b. VISUALIZACIÓN DEL LIMITBOX

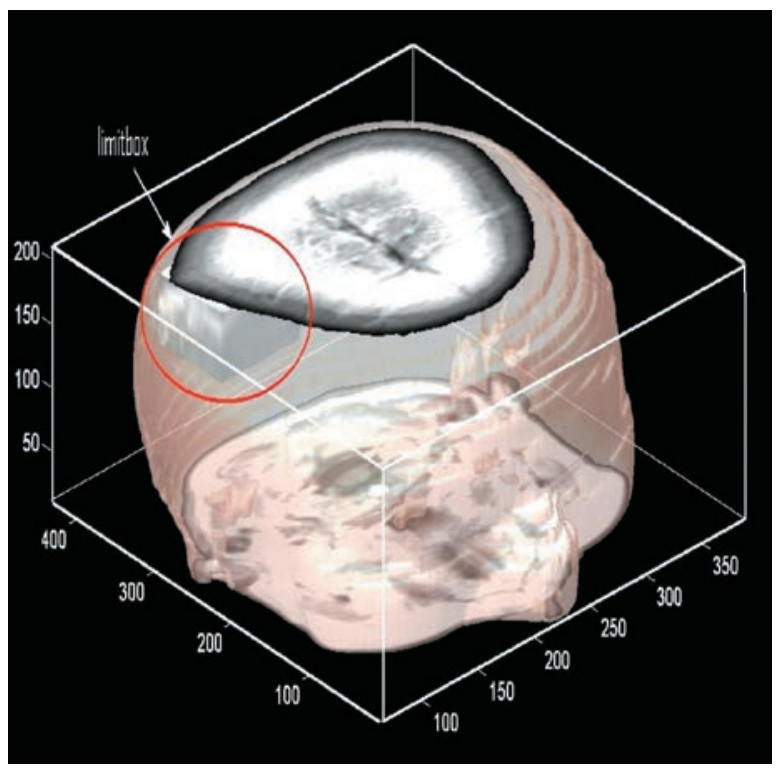

Fuente: autores.

Fig. 7. VISUALIZACIÓN DE LOS VOLÚMENES DELIMITADOS OJOS Y LESIÓN 1 Y 2

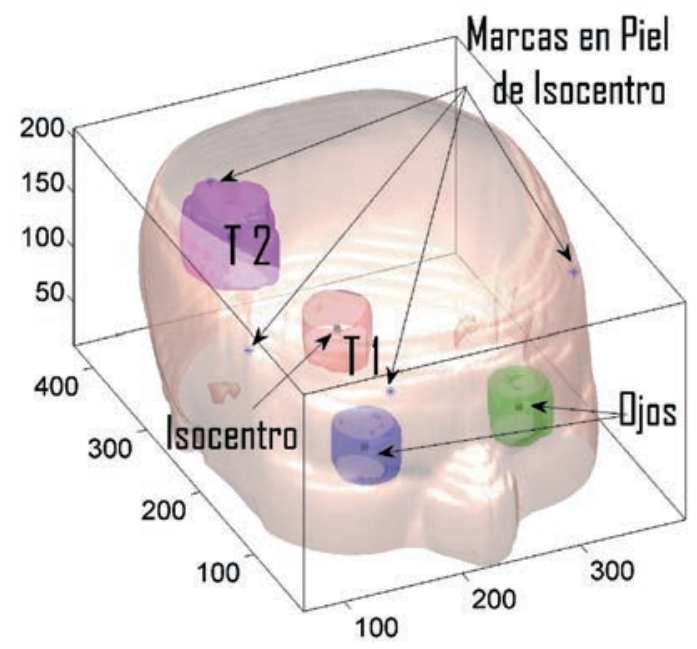

Fuente: autores. 
Fig. 8. VISUALIZACIÓN 3D DE LESIÓN TUMORAL Y SU CENTROIDE

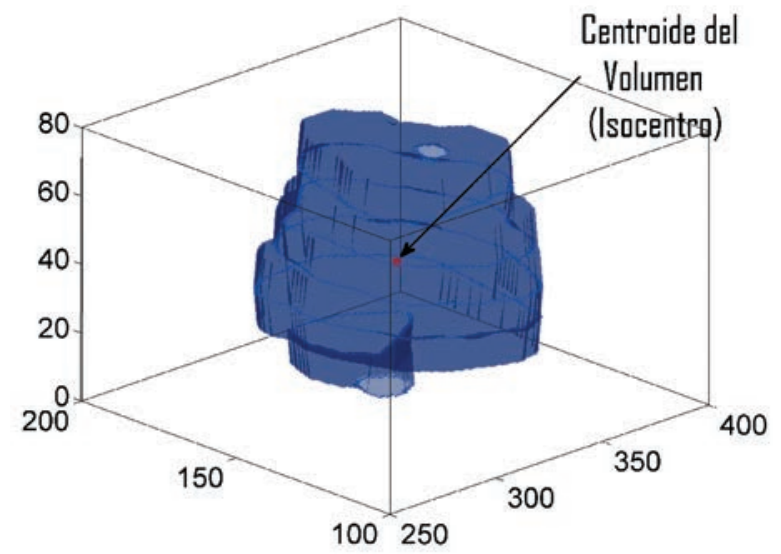

Fuente: autores.

Fig. 9a. EJES DE ROTACIÓN DEL EQUIPO DE TELETERAPIA SIN ANGULACIÓN

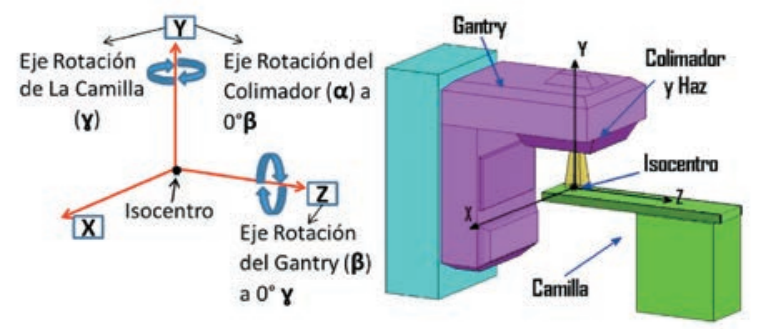

Fuente: autores.

Fig. 9b. CAMBIO EN MARCO DE REFERENCIA DEL EQUIPO POR ÁNGULOS $\gamma, \beta Y \alpha$

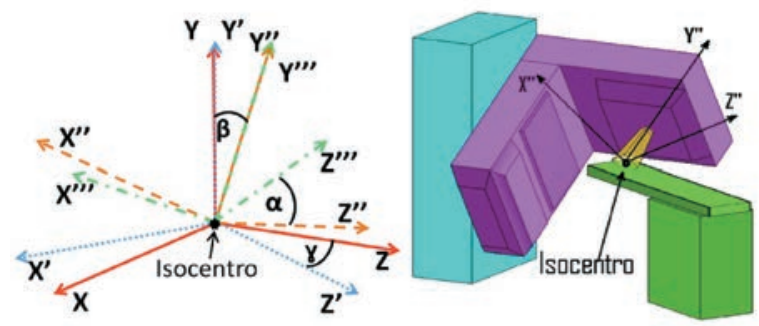

Fuente: autores.

El centro del volumen de la lesión o tumor representará siempre el punto de prescripción y la distancia entre cualquier punto de la piel a este, y será la profundidad a la cual se encuentra la lesión. Se redefine el punto centro de la lesión como el origen de nuestro sistema coordenado, representando así el punto de tratamiento. En términos clínicos este punto de tratamiento o centro de la lesión no debe presentar cambios de posicionamiento a la hora de realizar el tratamiento, debido a que es necesa- rio posicionar al paciente en el mismo lugar en que se tomaron las IDM y garantizar que este punto centro de la lesión esté ubicado espacialmente donde queda el punto Isocentro del equipo de tratamiento de teleterapia; este es el punto en el espacio donde se intersectan los ejes de rotación de la camilla, el gantry y el colimador (Fig. 9a).

\subsection{2. Ángulo de la camilla}

Al definir este punto de referencia es posible hacer incidir sobre él un número infinito de haces de tratamiento por lo cual es necesario acotar todas las posibilidades con respecto a las características morfológicas del volumen a tratar y al grupo de órganos visualizados, evitando al máximo irradiarlos sin ser necesario.

La camilla es la parte del equipo de tratamiento donde se posiciona el paciente. Guardando los parámetros de posicionamiento al momento de la toma de las IDM, la angulación de la camilla es independiente de las otras dos y es además el movimiento que permite realizar una exploración en 3D. Esta información no puede ser suministrada por un TPS en 2D, siendo esta una de las grandes ventajas de tener información 3D de las zonas afectadas (tumores, entre otros).

La rotación de la camilla permite girar al paciente en el plano xz con respecto al eje y, de tal forma que cualquier angulación ( $\mathrm{\gamma}$ ) de la camilla produce un cambio de las coordenadas de los puntos de cada volumen definido en los procesos anteriores, remitiéndolos a un nuevo marco de referencia $x^{\prime}, y^{\prime}$ y z', como se ve en la Fig. 9b.

\subsection{3. Ángulo del Gantry}

En muchos de los tratamientos no basta solo con mover la camilla, debe moverse también el Gantry. Esta parte del equipo es la que posee el sistema acelerador y el sistema productor de radiación ionizante [5]. El gantry gira en el plano $x^{\prime} y^{\prime}$ con respecto al eje $z^{\prime}$. De igual forma que el caso anterior, al girar el gantry un ángulo $(\beta)$, manteniendo el ángulo $\gamma$, se produce un nuevo cambio en el marco de referencia, pero este será dependiente del giro de la camilla y estará definido como un nuevo marco de referencia $x^{\prime \prime}, y^{\prime \prime}$ y z" (ver Fig. 9b). 


\subsection{4. Ángulo del colimador}

Este último sistema está ubicado en la parte superior del gantry y es la encargada de colimar la radiación producida por el equipo de tratamiento, restringiendo el área del haz de tratamiento. La radiación es producida en un punto dentro del gantry y al salir del equipo debe ser colimado con un sistema de bloques de tugsteno en forma rectangular, por lo cual la forma geométrica del haz es piramidal.

En algunos de los tratamientos en teleterapia es necesario girar el colimador para mejorar la irradiación de las zonas adyacentes, de tal forma que una disposición de campo en forma cuadrada puede cambiarse a un campo de forma de romboide, al girar el colimador un ángulo $(\alpha)$ en el plano $z^{\prime \prime} x^{\prime \prime}$ con respecto al eje y" y manteniendo las angulaciones $\beta$ y $\gamma$, el sistema coordenado resultante será x"', $y^{\prime \prime \prime}$ y z"', ver Fig. 9b.

\subsection{Parámetros de cálculo de tiempo de tratamiento}

Una vez establecidos los parámetros de simulación es posible definir con claridad los dos parámetros necesarios para el cálculo (profundidad y tamaño de campo), ya que sin una incidencia fija estos dos son inconclusos.

\subsubsection{Profundidad de tratamiento}

La definición de profundidad en el ámbito de la radioterapia se refiere a la distancia desde el punto de prescripción o isocentro del volumen a tratar, hasta el punto en piel por el cual entra el eje central del haz de tratamiento ver Fig. 10.

Fig. 10. DEFINICIÓN DE PROFUNDIDAD Y TAMAÑO DE CAMPO CON AYUDA DE LA VISUALIZACIÓN 3D EN LESIÓN 1

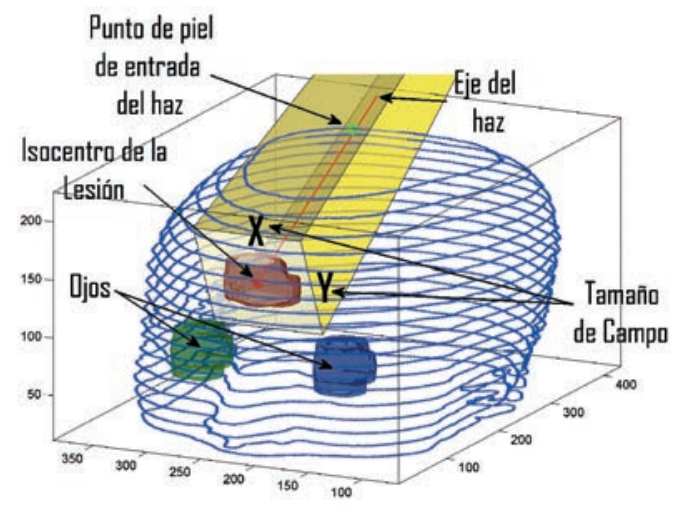

Fuente: autores.
Al definir la incidencia del haz, es posible establecer la profundidad, ya que tenemos la información de la coordenada del punto de piel por donde entra el haz, el cual es obtenido aplicando los procesos de rotación de los parámetros de simulación y las coordenadas del centro de la lesión o Isocentro, y aplicando la ecuación de distancia euclidiana (2) se obtiene un valor.

$$
d_{i}=\sqrt[2]{x_{i}^{2}+y_{i}^{2}+z_{i}^{2}}
$$

Este valor se está dando para cualquier angulación de las partes del equipo en forma tridimensional, brindando mayor información que los TPS en $2 \mathrm{D}$.

\subsubsection{Tamaño de campo}

Al describir el sistema de colimadores se menciona la forma piramidal del haz de tratamiento, donde la base de dicha pirámide representa el área de tratamiento o tamaño de campo, este se define a una distancia de $1 \mathrm{~m}$ del punto fuente de radiación en dirección del haz (acelerador). Este plano siempre estará en forma perpendicular a la dirección de incidencia del haz, de manera que el área del tumor que se puede visualizar en este plano da la información suficiente para reducir al máximo el tamaño del campo de tratamiento, de tal forma que el rectángulo que estructura el campo, contenga en su interior el área descrita por el volumen de lesión. Es necesario dejar un pequeño margen externo al contorno de la lesión [14], [15], [18] (Fig. 10), debido a posibles movimientos y la existencia de células tumorales en zonas aledañas.

\section{CÁlCULO A PUNTO}

El cálculo a punto consiste en la determinación del tiempo necesario para depositar una cantidad de energía en un punto definido, con unas condiciones restringidas por los parámetros de cálculo, los cuales a su vez son limitados al comportamiento de la radiación al interactuar con la materia [1],[18]. La forma de determinar el comportamiento de deposición de energía de los fotones al interactuar con la materia es posible con los datos de calibración del equipo de tratamiento con equipos detectores de radiación [19]. 
Los factores incluidos en el cálculo de tiempo de tratamiento para depositar una dosis determinada en el cuerpo, utilizando técnica de distancia fuente piel [18], [23] a una profundidad definida y con parámetros establecidos por el radioterapeuta y el físico son:

Dpres. La dosis de prescripción a un punto dentro de la zona tumoral o área de tratamiento, dada por el radioterapeuta.

D. La tasa de dosis o rendimiento del equipo; este factor es determinado por el proceso de calibración dosimetría absoluta, es la tasa de dosis estándar para el equipo.

(PDD) d, tc. El factor Define el porcentaje de dosis a una profundidad (d) y con un tamaño de campo (tc).

Sc (tc). Es la dispersión sufrida por el haz de radiación en el colimador; este efecto es producido por la interacción entre la radiación y el sistema de colimadores.

Fac SSD. Este factor representa el cambio en la dosis por el comportamiento de la radiación con la distancia.

Sp (tc). Representa el efecto de la dispersión que sufre la radiación dentro del cuerpo.

Estos factores son determinados por la dosimetría del equipo [24], [18] y son completamente dependientes de la profundidad y del tamaño de campo. De forma inversa, teniendo el tiempo de tratamiento, el tamaño de campo y la profundidad de cada uno de los órganos, se puede brindar la información de la dosis recibida por estos en eltratamiento. Los aceleradores lineales utilizados en teleterapia son usualmente calibrados para dar una tasa de dosis $1 \mathrm{rad}$ (1 cGy /UM) [6], [23], como es el caso del acelerador utilizado; este valor es definido en la profundidad de referencia $5 \mathrm{~cm}$, para un tamaño de campo de $10 \times 10 \mathrm{~cm}$ y una distancia fuente-superficie de $100 \mathrm{~cm}$.

El cálculo del tiempo de tratamiento para técnica SSD se define como unidades monitor (UM) para el acelerador (3) [22] (pg162,(ec 10-8)), datos necesarios para entregar una cantidad de dosis $\mathrm{D}$ al punto centro del tumor, a una profundidad d para un tamaño de campo tc, que está dado por:

$$
U M=\frac{D_{\text {pres }} * 100}{\widetilde{D} * P D D_{d, t c} * S_{c}(t c) * S_{p}(t c) * F a c S S D}
$$

El tiempo de tratamiento en el acelerador por tratarse de un equipo electrónico es dependiente del flujo eléctrico, y como este no es constante es necesario referirlo como unidades de radiación medidas por un sistema monitor (cámaras de ionización) ubicado cerca al punto de origen del haz, dando información de la radiación que sale del equipo.

\section{DISCUSIÓN Y RESULTADOS}

En este trabajo se creó una interfaz que permite ver un ambiente en 3D que incluye una simulación de equipo de tratamiento y también del haz de tratamiento incidiendo sobre el volumen del cuerpo. En el ámbito clínico la incidencia de haz de tratamiento como el número de haces utilizados es definido por el físico y el radioterapeuta, según su experiencia y los protocolos establecidos. En la mayoría de los casos, los tratamientos son administrados haciendo uso de más de un haz, por este motivo, la interfaz gráfica creada permite mover el haz de tratamiento de forma manual (sin invadir al paciente), dejando definir las mejores opciones de ataque o incidencia del haz, de forma que se evite al máximo la irradiación de los órganos definidos y así poder extraer los tres ángulos del equipo de tratamiento, utilizando como ayuda visual la reconstrucción en 3D resultante del procesamiento de las IDM.

A las IDM se les realizó medidas de profundidad (desde la piel a la zona afectada en el cuerpo del paciente), girando el gantry en dirección opuesta al sentido horario y fijando el punto de interés en el Isocentro de la lesión (como se ve en la Fig. 10). En la Fig. 11 se muestran los datos de las mediciones con el TPS Kenos 2D y los datos extraídos de acuerdo al método explicado en esta sección. Se puede observar que el comportamiento de las medidas es similar. Al realizar la comparación entre los datos del TPS Kenos 2D y los del método implementado, la desviación estándar residual es $0.326 \mathrm{~mm}$. 
Fig. 11. DATOS RESULTANTES DE MEDICIÓN DE DISTANCIA EN EL PLANO DEL ISOCENTRO

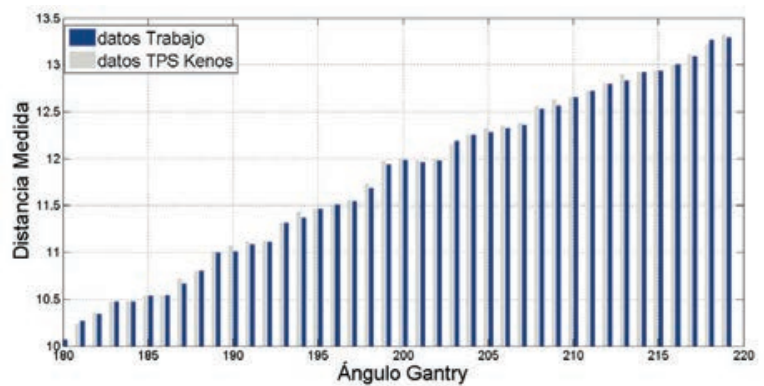

Fuente: autores.

Este tipo de visualización en 3D brinda una gran información, ya que permite escoger el menor tamaño de campo de una cantidad de ángulos de ataque, brindando un mayor beneficio que los TPS en 2D.

Una vez se obtienen los parámetros de simulación de entorno y de cálculo de tiempo de tratamiento, es posible obtener el tiempo de tratamiento por sesión de acuerdo a las especificaciones del radioterapeuta, como son: la dosis a suministrar y el número de fracciones necesarias según el diagnóstico.

En este trabajo solo se utiliza los datos de dosimetría (absoluta y relativa) de rayos X de $6 \mathrm{MV}$, producidos por un Acelerador lineal Clinac 6Ex de Varian de propiedad de Radioterapia del Norte Ltda.

Teniendo como base los datos de calibración dosimétrica (absoluta y relativa) [20],[21] del Acelerador lineal, restringidos a los valores de profundidad y de tamaño de campo que fueron obtenidos por el procesamiento de imágenes y los tipos de cálculo de dosis a punto [22],[23] en tratamientos de radioterapia, y prescribiendo una dosis cualquiera, se tienen las herramientas necesarias para determinar el tiempo de tratamiento por fracción.

Con los datos de profundidad obtenidos en la etapa de procesamiento y los equivalentes en el TPS se realizó el cálculo de las UM, los resultados se muestran en la Fig. 12. La comparación entre los datos del TPS Kenos 2D y los del trabajo muestran una desviación estándar residual de 0.738 UM. Es de aclarar que esta comparación no se realiza con el fin de evaluar el TPS Kenos, todo lo contrario, esto permite evaluar el método descrito.
Fig. 12. DATOS DE PROFUNDIDAD VS CÁLCULO A PUNTO PARA UNA DOSIS DE PRESCRIPCIÓN 5OCGY Y TAMAÑO DE CAMPO DE 10X10CM

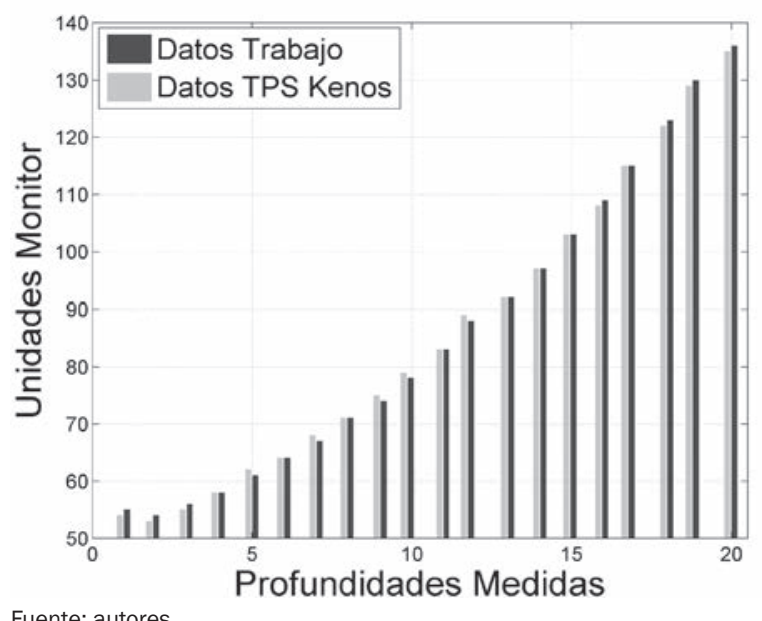

Fuente: autores.

\section{CONCLUSIONES}

En el área de la salud como en radioterapia es muy importante este tipo de ayudas visuales, ya que garantizan un mejor tratamiento y brindan un gran número de posibilidades de modificaciones en los tratamientos, siendo de mejor ayuda las imágenes en 3D. El alto costo de los sistemas de simulación y planeación utilizados en esta área, puede disminuirse con la implementación de herramientas de este tipo que pueden ser desarrolladas en el país, reduciendo los costos de equipos utilizados y brindando una garantía a los tratamientos; la investigación en el área de radioterapia es muy joven en el país.

Los datos de tamaño de campo son subjetivos, ya que es el radioterapeuta o el físico encargado quienes definen los valores según su experiencia y el diagnóstico del paciente, como de la habilidad a la hora de definir el área o volumen de la lesión.

El trabajo es sintetizado en una interfaz gráfica amigable que permite realizar todos estos procesos de una forma sencilla, respaldada por herramientas matemáticas que permitan determinar parámetros de simulación de entorno y un posible tiempo de tratamiento por fracción para tratamiento en radioterapia y dosis en los órganos o tejidos de interés, además puede ser utilizado como herramienta en reconstrucción tridimensional del cuerpo (atlas humano 3D) para diseño y modelación computacional 3D. 


\section{AGRADECIMIENTOS}

Los autores quieren agradecer a los centros de tratamiento de cáncer: Radioterapia del Norte Ltda. e Instituto de Cancerología de Medellín y también a los centros de diagnóstico: Clínica Santa Ana y Hospital Erasmo Meoz por las Imágenes facilitadas para este trabajo. Los datos de dosimetría absoluta y relativa fueron de libre acceso ofrecidos por Radioterapia del Norte Ltda. Martha Lucía Molina Prado y Néstor Alonso Arias Hernández agradecen a Colciencias y a la Universidad de Pamplona.

\section{REFERENCIAS}

[1] W. E. Burcham, "Física Nuclear", $2^{\text {nd }}$ ed. Barcelona, España: Ed. Reverté, 2003, cap.1-5, pp. 47-153.

[2] C. S. Pedrosa,R. Casanova, "Monografía de Diagnóstico por imágenes”. Madrid, España: Mc Graw-Hill, 1991, pp. 13-160.

[3] G. Passariello, F. Mora, “Imágenes Médicas: Adquisición, Análisis, Procesamiento e Interpretación. Miranda, Venezuela: Universidad Simón Bolívar, 1995, pp. 2-47.

[4] P. Sánchez Galiano, "Introducción a la física de la radioterapia". Viena, Austria: Hospital Central de Asturias, Unidad de Radiofísica, 2005, pp. 1-72.

[5] H. Ziessman, JP. OMalley, "Medicina Nuclear (los requisitos en radiología)", $3^{\text {th. }}$ ed. Madrid, España: Elsevier, 2007, pp. 52-158.

[6] Varian Medical System, "Series Clinic Acelerator System Basics”, Palo Alto California, USA: Varian, 1998.

[7] J. P. Gunilla, "Manual de Física de la Radioterapia (La teleterapia)”. Barcelona, España: Masson, 1996, pp. 1-60.

[8] Organismo Internacional de Energía Atómica, “Determinación de la Dosis absorbida en haces de fotones y electrones en Agua (Código de Práctica Internacional), $2^{\text {nd }}$ ed. Viena, Austria: Colección de Informes técnicos, no. 277,1998 , pp. 1-76.

[9] R. González and R. E. Woods, "Tratamiento Digital de Imágenes", $3^{\text {th. }}$ ed. Delaware, USA: Wilmington. Addison-Wesley Libero America S.A,1998, cap.1-4 .

[10] J. Esquela Elizondo and L. E. Palofox. "Fundamentos de Procesamiento de Imagen". USA: Conatec 2002, Universidad Autónoma de Baja California, 2002, pp. 1-49.

[11] A. C. Kak. "Principles of Computerized tomographic Imaging", $5^{\text {th }}$ ed. Philadelphia, PA, USA: Electronic Copy by A.C. Kak and Malcom Staney, IEEE Pres, 1999, cap.1-7, pp. 5-110, 276-292.
[12] J. G. Proakis, "Digital Signal Processing", $3^{\text {th }}$ ed. Los altos California, USA: Pearson Prentice Hall, 2007, pp. 2-95.

[13] J. Canny, "A Computational Approach to Edge Detection", IEEE Trans. Pattern Analysis and Machine Intelligence, vol.PAMI-8, Issue 6, 1986, pp. 679-698.

[14] C. Perez, and L. Brady. "Perez and Brady's Principles and Practice of Radiation Oncology", $5^{\text {th }}$ ed. Philadelphia, USA: Lippincott Williams \& Wilkins, 2008, cap.1-10, pp 76-215.

[15] International Commission on Radiation Units and Measurements, "Prescripción, Registro y Elaboración de Informes en la Terapia con Haces de Fotones (Versión en Español de la Sociedad Española de Física Médica), reporte ICRU, no. 50, 1993.

[16] J. Dobbs, A. Barrett and D. Ash, "Practical Radiotherapy Planning", $3^{\text {th }}$ ed. New York, USA: New York. Arnold, 1992, cap.1-4, pp. 1-33.

[17] H. Vélez y W. Rojas. Radiología e Imágenes Diagnósticas, $2^{\text {th }}$ ed. Medellin: Corporación para Investigaciones Biológicas, 2007.

[18] E. Podgorsak, "Review of Radiation Oncology Physics: A handbook for teacher and students". Montreal, Canada: Department of Medical Physics Mc Gill, University Healt Center, 2003, pp. 249-316.

[19] Instituto de Geología y Minas (Colombia), "Curso de Protección Radiológica para el manejo de Material Radioactivo", Bogotá,Colombia: Grupo de Seguridad Nuclear y Radioprotección, 2002.

[20] International Atomic Energy Agency (IAEA, Absorbed Dose Determination in External Beam Radiotherapy (An International Code of practice for dosimetry Based on Standards of Absorved Dose to Water), "Tecnical Reports Series", no. 398, 2000.

[21] F. H. Attix, "Introduction of Radiological Physicis and Radiation Dosimeter". Madison Wisconsin, USA: John Wiley \& Sons, 1986, pp. 203-290.

[22] F. M. Khan, "The Physics of Radiation Therapy, $4^{\text {th }}$ ed. Baltimore: Ed. Lippincott williams \& wilkins, 2010, pp. 26-95,158-175; ec, pp.162; ec, pp. 10-8.

[23] Gunilla C. "Radiation Therapy Planning", $2^{\text {nd }}$ ed. Durham, USA: Ed. Durham, McGraw-Hill, 1992, cap.1-3, pp. 32-159.

[24] O. Bernal y S. Martínez, “Dosimetría para Campos Asimétricos”. Tunja: Universidad Pedagógica y Tecnológica de Colombia, 2004.

[25] E. Barbará, R. Sánchez, E. González, “Comparación de Algoritmos de Segmentación de Ruido Aplicados a Imágenes de Resonancia Magnética", Revista Electrónica, Automática y Comunicaciones (RIELAC). vol. 33, no, 3, 2012. 